strategies for secondary metabolite yield improvement in Actinomycetes. Antonie van Leeuwenhoek 79:251-259.

5.Rodriguez, E. and R. McDaniel. 2001. Combinatorial biosynthesis of antimicrobials and other natural products. Curr. Opin. Microbiol. 4:526-534.

6.Mordarska, H., S. Cebrat, B. Blach, and M. Goodfellow. 1978. Differentiation of nocardioform Actinomycetes by lysozyme sensitivity. J. Genet. Microbiol. 109:381-384.

7.Kutchma, A.J., M.A. Roberts, D.B. Knaebel, and D.L. Crawford. 1998. Small-scale isolation of genomic DNA from Streptomyces mycelia or spores. BioTechniques 24: 452-456.

8.Assaf, N.A. and W.A. Dick. 1993. Spheroplast formation and plasmid isolation from Rhodococcus spp. BioTechniques 15:10101012

9.Rao, R.N., M.A. Richardson, and S. Kuhstoss. 1987. Cosmid shuttle vectors for cloning and analysis of Streptomyces DNA. Methods Enzymol. 153:166-198.

10.Lee, Y.K., Kim, H.W., Liu, H.K. and H.K. Lee. 2003. A simple method for DNA extraction from marine bacteria that produce extracellular materials. J. Microbiol. Methods 52: 245-250.

11.Ogawa, H., S. Imai, A. Satoh, and M. Kojima. 1983. An improved method for the preparation of Streptomycetes and Micromonospora protoplasts. J. Antibiot. 36:184-186.

12.Ezaki, T. and S. Suzuki. 1982. Achromopeptidase for lysis of anaerobic gram positive cocci. J. Clin. Microbiol. 16:844-846.

13.Leonard, R.B. and K.C. Carroll. 1997. Rapid lysis of gram positive cocci for pulse field electrophoresis using achromopeptidase. Diagn. Mol. Pathol. 6:288-291

14.Li, S., S. Norioka, and F. Sakiyama. 1997. Purification, staphylolytic activity, and cleavage sites of a-lytic protease from Achromobacter lyticus. J. Biochem. 122:772-778

15.Yanagida, T. and H. Ogawara. 1980. Deoxyribonucleases in Streptomyces. J. Antibiot. 33:1206-1207.

16.Sanchez, J., C. Barbes, A. Hernandez, C.R. de los Reyes-Gavilan, and C. Hardisson. 1985. Restriction-modification systems in Streptomyces antibioticus. Can. J. Microbiol. 31:942-946

17.de los Reyes-Gavilan, C.G., J.F. Aparicio, C. Barbes, C. Hardisson, and J. Sanchez. 1988. An exocytoplasmic endonuclease with restriction function in Streptomyces antibioticus. J. Bacteriol. 170:1339-1345

Received 16 July 2003; accepted 11 September 2003.

Address correspondence to Jo-Anne Chuck, School of Science, Food and Horticulture, Parramatta Campus, University of Western Sydney, Locked Bag 1797, Penrith South DC 1797 NSW, Australia. e-mail: j.chuck@uws.edu.au

\title{
Improved transfection technique for adherent cells using a commercial lipid reagent
}

\author{
Joel Escobedo and Timothy J. Koh \\ University of Illinois at Chicago, Chicago, IL, USA
}

BioTechniques 35:936-940 (November 2003)

A variety of techniques have been developed for cell transfection including chemical [e.g., calcium phosphate (1) and lipid-based methods (2)], physical [e.g., electroporation (3)], and viral [e.g., retrovirus (4)] approaches. Nonviral techniques tend to be relatively safe and simple but also tend to be relatively inefficient compared with viral techniques. Lipid-based reagents, including liposomal and nonliposomal lipids, have become increasingly popular for in vitro and in vivo gene transfer (5). Despite the success of lipid-based methods, existing approaches may not be sufficient when an experiment requires transfection of the majority of cells in a population. We have developed an improved method using a commercial nonliposomal lipid reagent (Effectene $^{\mathrm{TM}}$; Qiagen, Valencia, CA, USA) to transfect cultured adherent cells that results in improved transfection efficiencies. We transfected C2C12 skeletal myoblasts and NIH-3T3 fibroblasts immediately after trypsinization, while the cells were in suspension; the standard approach is to transfect adherent cells several hours after they have attached to the culture dish. The transfection efficiency of the new method (70\%-80\% of cells transfected) may obviate the need for time-consuming stable transfections in many situations.

For the standard transfection procedure, cells were transfected following attachment to plastic culture dishes according to the manufacturer's protocol. C2C12 myoblasts and NIH-3T3 fibroblasts were obtained from American Type Culture Collection (Manassas, VA, USA). Cells were seeded at $2 \times$ $10^{5}$ cells per well in 6 -well plates in $2 \mathrm{~mL}$ of growth medium composed of Dulbecco's modified Eagle's medium (DMEM; Sigma, St. Louis, MO, USA) supplemented with $10 \%$ fetal bovine serum and $1 \%$ penicillin/streptomycin (GIBCO/Invitrogen, Grand Island, NY, USA). Cells were incubated overnight at $37^{\circ} \mathrm{C}$ and $5 \% \mathrm{CO}_{2}$. The following morning, lipid-DNA complexes were prepared according to manufacturer's instructions using a 1:8 DNA-to-En-

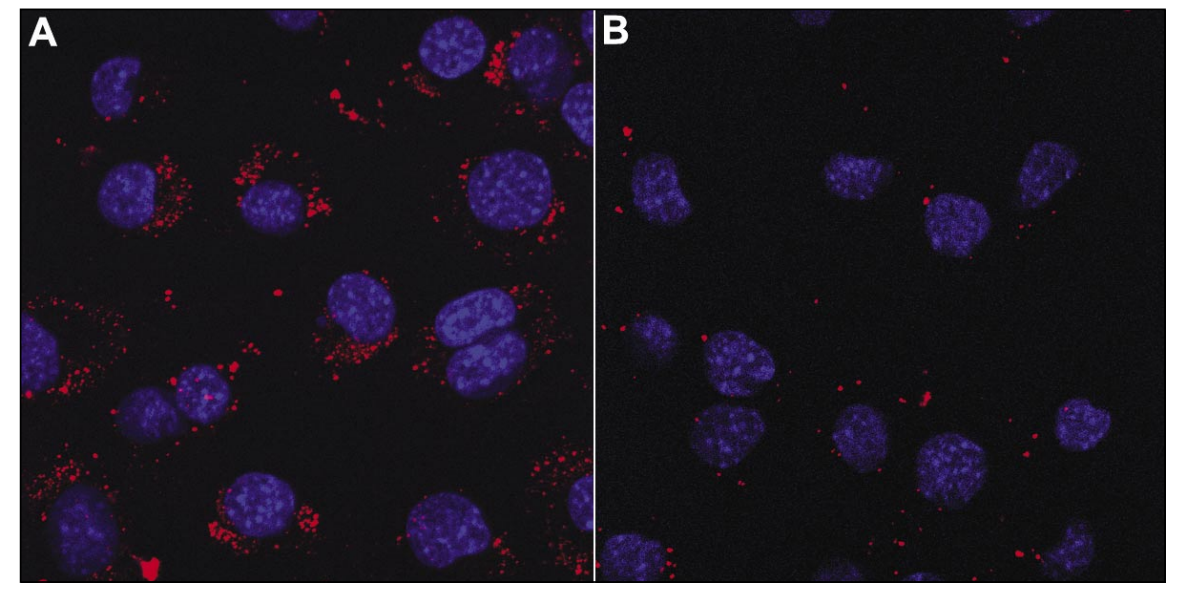

Figure 1. C2C12 myoblasts transfected with labeled plasmid DNA. C2C12 myoblasts transfected with rhodamine-labeled plasmid DNA showed greater DNA uptake when using the new transfection procedure (A) compared to the standard procedure (B). For both procedures, $2 \times 10^{5}$ cells were transfected with $1 \mu \mathrm{g}$ DNA, and cells were viewed using confocal microscopy $24 \mathrm{~h}$ after transfection. 
hancer ratio and a 1:15 DNA-to-Effectene ratio. DNA was incubated with Enhancer in DNA Condenser buffer for $2 \mathrm{~min}$ at room temperature to condense the DNA, and then Effectene reagent was added and the mixture incubated for a further $10 \mathrm{~min}$ at room temperature to allow lipid-DNA complex formation. During this incubation period, cell growth medium was replaced with fresh medium. Growth medium was then added to the complexes to a final volume of $600 \mu \mathrm{L}$, and the complexes were added to the cells. Cells were incubated with lipid-DNA complexes

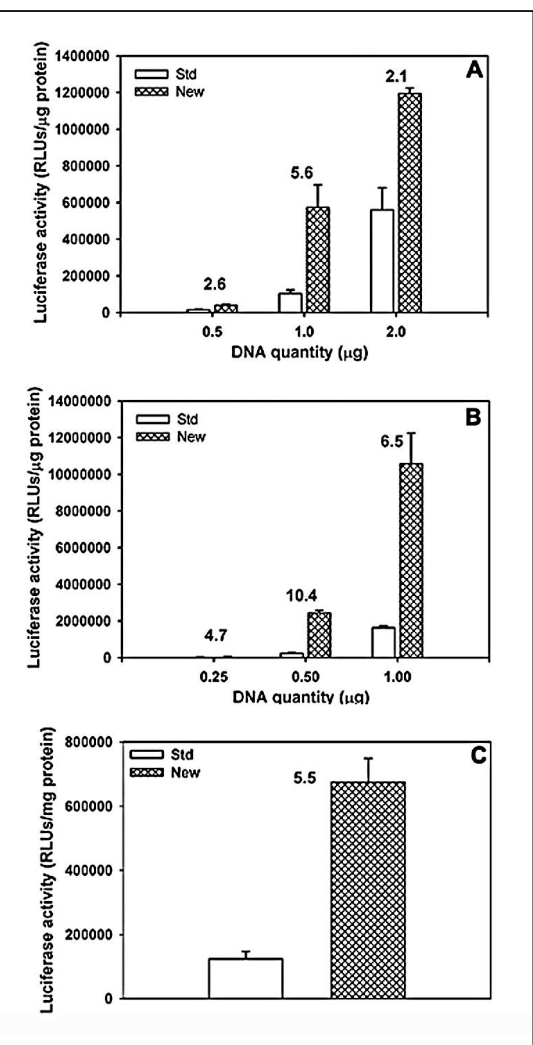

Figure 2. C2C12 and NIH-3T3 cells transfected with luciferase expression vectors. $\mathrm{C} 2 \mathrm{C} 12$ myoblasts (A) and NIH-3T3 fibroblasts (B) transfected with luciferase expression vectors showed up to 10 -fold greater luciferase activity using the new procedure compared with the standard procedure. For both procedures, $2 \times 10^{5}$ cells were transfected with varying amounts of DNA and luciferase activity was measured $48 \mathrm{~h}$ after transfection. For assessing expression in $\mathrm{C} 2 \mathrm{C} 12$ myotubes (C), 2 $\times 10^{5}$ myoblasts were transfected with $1 \mu \mathrm{g}$ DNA, grown to confluence, and differentiated for 4 days, and then luciferase activity was measured. Bars represent mean \pm standard errors $(\overline{\mathrm{X}} \pm \mathrm{SEM} ; n=$ 4). Results are representative of two independent experiments. Numbers above bars indicate fold increase in transfection efficiency using new procedure compared with standard procedure. for $24 \mathrm{~h}$ at $37^{\circ} \mathrm{C}$ and $5 \% \mathrm{CO}_{2}$, and then complexes were removed and fresh growth medium added. For experiments testing vector expression in myoblasts and fibroblasts, cells were collected 24 or $48 \mathrm{~h}$ posttransfection. For experiments testing expression in myotubes, the medium was switched to differentiation medium (DMEM $+2 \%$ horse serum) when cells reached confluency, and cells were allowed to differentiate for 4 days with daily changes in medium before collection.

For the new procedure, cells were transfected immediately after trypsinization and replating. Lipid-DNA complexes were prepared using a 1: 8 DNA-to-Enhancer ratio and a 1:15 DNA-to-Effectene ratio, identical to the standard procedure. Myoblasts or fibroblasts grown in $10-\mathrm{cm}$ dishes to $70 \%$ confluency in growth medium were trypsinized and then seeded at 2 $\times 10^{5}$ cells per well in 6 -well plates in $2 \mathrm{~mL}$ of growth medium. Immediately after plating cells, while the cells were still in suspension, growth medium was added to the complexes to a final volume of $600 \mu \mathrm{L}$, and the complexes were added to the cells. Cells were incubated with lipid-DNA complexes while they attached to the culture dish at $37^{\circ} \mathrm{C}$ and $5 \% \mathrm{CO}_{2}$, and then complexes were removed after $24 \mathrm{~h}$ and fresh growth medium was added. Cells were either collected at 24 or 48 h posttransfection or after 4 days of myotube differentiation.

The new transfection procedure resulted in substantially greater uptake of rhodamine-labeled plasmid DNA (Gene Therapy Systems, San Diego, CA, USA) compared with the standard procedure as assessed by confocal microscopy (Figure 1). In these images, nuclei were stained with Hoechst 33258. For both new and standard transfection procedures, the majority of labeled DNA was observed in a punctate vesicle-like pattern, suggesting that DNA may have entered the cell by endocytosis. Previous investigations have supported a primary role for endocytosis in uptake of lipid-complexed DNA (6-8).

When transfecting a cytomegalovirus (CMV)-luciferase vector (Gene Therapy Systems), the new procedure resulted in up to 10-fold greater luciferase activity compared with the standard procedure (Figure 2). The magnitude of the increase in luciferase activity was

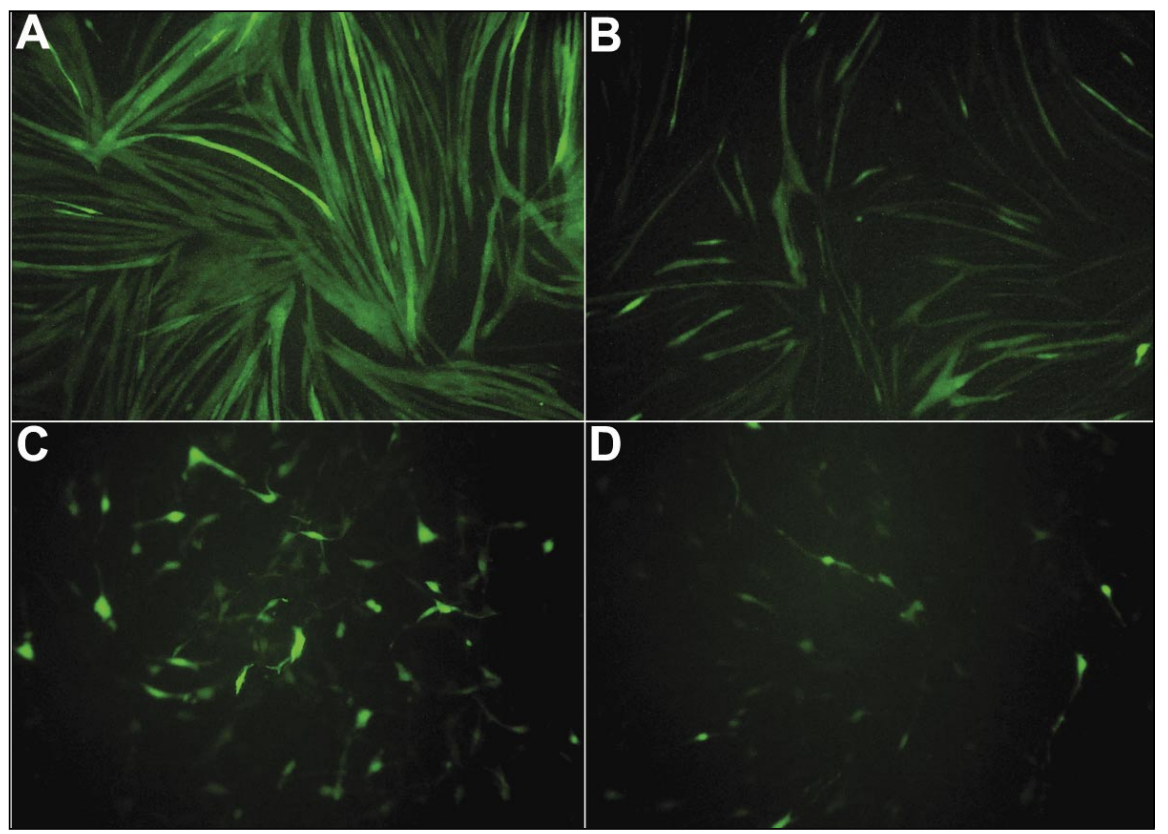

Figure 3. C2C12 and $\mathrm{NIH}-3 \mathrm{~T} 3$ cells transfected with green fluorescent protein (GFP) vectors. $\mathrm{C} 2 \mathrm{C} 12$ myotubes (A and B) and NIH-3T3 fibroblasts (C and D) transfected with GFP expression vectors showed a greater percentage of cells expressing GFP using the new transfection procedure (A and C) compared with the standard procedure (B and D). For C2C12 myotubes, $2 \times 10^{5}$ cells were transfected with $1 \mu \mathrm{g}$ DNA, grown to confluence, and differentiated for 4 days, and then images were recorded. For NIH-3T3 cells, $2 \times 10^{5}$ cells were transfected with $0.5 \mu \mathrm{g}$ DNA and images recorded $24 \mathrm{~h}$ after transfection. 
cell type- and DNA dose-dependent. For $\mathrm{C} 2 \mathrm{C} 12$ cells, the largest increase in luciferase activity (approximately 6-fold) was achieved using $1 \mu \mathrm{g}$ DNA. For NIH-3T3 cells, the largest increase in luciferase activity (approximately 10-fold) was achieved using $0.5 \mu \mathrm{g}$ DNA. When $\mathrm{C} 2 \mathrm{C} 12$ myoblasts were transfected with $1 \mu \mathrm{g}$ DNA, grown to confluence, and then differentiated for 4 days, the new transfection procedure resulted in an approximately 6-fold increase in luciferase activity compared with the standard procedure. These results indicate that the increased transfection efficiency associated with the new procedure is maintained for at least 6 days after transfection and is maintained throughout differentiation of $\mathrm{C} 2 \mathrm{C} 12$ cells.

Using the new transfection procedure to introduce green fluorescent protein (GFP) expression vectors into $\mathrm{C} 2 \mathrm{C} 12$ and NIH-3T3 cells resulted in a substantially greater number of cells expressing detectable levels of GFP in C2C12 myotubes and NIH-3T3 fibroblasts (Figure 3 ). In the figures shown, C2C12 myoblasts were transfected with $1 \mu \mathrm{g}$ DNA, grown to confluence, and then differentiated for 4 days, and NIH-3T3 cells were transfected with $0.5 \mu \mathrm{g}$ DNA and were approximately $50 \%$ confluent when imaged. Qualitative assessments of transfection efficiencies by two independent observers ranged from $10 \%-20 \%$ for the standard procedure and $70 \%-80 \%$ for the new procedure for both NIH$3 \mathrm{~T} 3$ and $\mathrm{C} 2 \mathrm{C} 12$ cells. The transfection efficiencies achieved with the new procedure may be sufficient for many experiments that previously required viral transfection or time-consuming stable transfections.

Our procedure was also effective for introducing an overexpression vector to increase expression of the small heat shock protein $\mathrm{HSP} 25$ in $\mathrm{C} 2 \mathrm{C} 12$ myotubes and for introducing phosphorothioate antisense oligonucleotide into $\mathrm{C} 2 \mathrm{C} 12$ myoblasts to knock down constitutive levels of HSP25. Using the new procedure to introduce $1 \mu \mathrm{g}$ of a HSP25 expression vector into $\mathrm{C} 2 \mathrm{C} 12$ cells resulted in an approximately $80 \%$ increase in HSP25 levels above levels observed following transfection of an empty vector, whereas the standard procedure resulted in only an approximately $30 \%-40 \%$ increase in HSP 25 . Using this procedure to introduce $1 \mu \mathrm{g}$ of antisense oligonucleotide against HSP25 (TriLink BioTechnologies, San Diego, CA, USA) into C2C12 cells resulted in an approximately $45 \%$ decrease in HSP25 levels compared with cells treated with transfection reagent only and cells transfected with a sense oligonucleotide construct, whereas the standard procedure resulted in little to no change in HSP25 levels. These results demonstrate the utility of the new transfection technique for introducing overexpression vectors and antisense oligonucleotides to modulate levels of constitutively expressed cellular proteins.

In summary, transfecting adherent cells immediately after trypsinization and replating improved transfection efficiency in $\mathrm{C} 2 \mathrm{C} 12$ muscle cells and NIH-3T3 fibroblasts up to 10 -fold. This new transfection procedure was also more effective in modulating levels of a constitutively expressed heat shock protein, HSP25, using overexpression vectors and antisense oligonucleotides. These findings are immediately useful for improving transfection efficiency of adherent cultured cells, and the transfection efficiencies achieved may obviate the need for time-consuming stable transfections in many situations. Further investigation into the mechanisms of improved transfection may reveal strategies to increase transfection efficiency in vivo.

\section{ACKNOWLEDGMENTS}

This work was supported by research grants from the Whitaker Foundation and TriLink BioTechnologies. J.E. was supported by a Postdoctoral Fellowship from the American Heart Association. Dr. Mathias Gaestel, Martin Luther University, provided the HSP25 expression vector, and Dr. Anne Knowlton, University of California, Davis, provided the antisense sequence for HSP25.

\section{REFERENCES}

1.Graham, F.L. and A.J. van der Eb. 1973. A new technique for the assay of infectivity of human adenovirus 5 DNA. Virology 52: 456-467.
2.Felgner, P.L., T.R. Gadek, M. Holm, R. Roman, H.W. Chan, M. Wenz, J.P. Northrop, G. M. Ringold, and M. Danielsen. 1987. Lipofection: a highly efficient, lipid-mediated DNA-transfection procedure. Proc. Natl. Acad. Sci. USA 84:7413-7417.

3.Neumann, E., M. Schaefer-Ridder, Y. Wang, and P.H. Hofschneider. 1982. Gene transfer into mouse lyoma cells by electroporation in high electric fields. EMBO J. 1:841-845.

4.Wolff, J.A., J.K. Yee, H.F. Skelly, J.C. Moores, J.G. Respess, T. Friedmann, and H. Leffert. 1987. Expression of retrovirally transduced genes in primary cultures of adult rat hepatocytes. Proc. Natl. Acad. Sci. USA 84:3344-3348

5.Armeanu, S., J. Pelisek, E. Krausz, A. Fuchs, D. Groth, R. Curth, O. Keil, J. Quilici, et al. 2000. Optimization of nonviral gene transfer of vascular smooth muscle cells in vitro and in vivo. Mol. Ther. 1:366-375

6.Wrobel, I. and D. Collins. 1995. Fusion of cationic liposomes with mammalian cells occurs after endocytosis. Biochim. Biophys. Acta 1235:296-304.

7.Zabner, J., A.J. Fasbender, T. Moninger, K.A. Poellinger, and M.J. Welsh. 1995. Cellular and molecular barriers to gene transfer by a cationic lipid. J. Biol. Chem. 270:1899719007.

8.Pires, P., S. Simoes, S. Nir, R. Gaspar, N. Duzgunes, and M.C. Pedroso de Lima. 1999. Interaction of cationic liposomes and their DNA complexes with monocytic leukemia cells. Biochim. Biophys. Acta 1418: 71-84.

Received 4 August 2003; accepted 19 September 2003.

Address correspondence to Timothy J. Koh, School of Kinesiology, University of Illinois at Chicago, 901 W. Roosevelt Road, Chicago,IL 60608,USA.e-mail: tjkoh@uic.edu 